\title{
TEORÍA LEGA DE SABIDURÍA EN ADULTOS Y RELACIONES CON EL YO IDEAL
}

\author{
V. Pelechano \\ P. González-Leandro \\ Universidad de La Laguna (Tenerife)
}

\section{RESUMEN}

300 adultos españoles, ambos géneros, con media de edad de 23,5 años cumplimentaron una prueba sobre teoría implícita de sabiduría (con inclusión de términos tales como inteligencia, sabio, anciano) y propuesta sobre yo ideal. Se llevó a cabo un análisis de conglomerados jerárquicos con dos agrupaciones significativas y rotacion varimax de las mismas. Inteligencia y sabiduría aparecen juntas en todos los análisis. Los resultados presentan algunas diferencias respecto a los de Takahashi y Bordia (2000). Un conglomerado agrupa aspectos relacionados con eficacia socio-laboral; $y$ el otro, con eficacia interpersonal (humanismo). El análisis evolutivo muestra una mayor importancia del primero con el incremento de edad (>30 años). El yo ideal se asocia con inteligencia, comprensión y sentido del humor en todo el rango de edad, mientras que "sabio" se asocia solamente con el yo ideal de manera tenue y a partir de los 30 años.

Palabras clave: TEORIA LEGA DE SABIDURÍA, INTELIGENCIA Y SABIDURIA, IMAGEN DEL YO IDEAL Y SABIDURIA.

Este trabajo ha sido hecho gracias al proyecto BS02002-06696 subvencionado por la Comisión Interministerial sobre Ciencia y Tecnología (Madrid). Las opiniones vertidas en este trabajo no comprometen a la entidad subvencionadora. Una versión reducida de este trabajo se presentó en el 9th European Congress of Psychology (2005).

Correspondencia: vpelecha@ull.es. 


\section{SUMMARY}

300 Spanish adults (mean of 23,5 years old, both genders) fulfilled the Takahashi and Bordia (2000) list on lay theory on wisdom and the concept of ideal self. Cluster analysis gave two significant nuclei: efficacy in work contexts and humanism. Intelligence and wisdom are perceived as a single cluster before and after 30 years old. Developmental analysis on ideal self shows strong associations with intelligent, comprehensive and humor sense. Wisdom appears to be relevant and has personal meaning (related to ideal-self) only after 30 years old.

\section{Key words: LAY-THEORY OF WISDOM, INTELLIGENCE AND WISDOM, IDEAL} SELF AND WISDOM.

Al menos tres tradiciones distintas dentro de la psicología se han ocupado en el estudio de la sabiduria y se han mantenido bastante independientes entre si hasta fechas muy recientes. La primera ha tenido que ver con la conversión de la psicología evolutiva en una psicología del ciclo vital (en la que se incluían los ancianos como última fase evolutiva) a partir de los trabajos pioneros, entre otros, de Erikson y el reconocimiento de los límites del acercamiento piagetiano en el estudio de los procesos cognitivos (el denominado "período post-formal" era demasiado largo e inespecífico). La segunda, proviene de los estudios sobre la inteligencia y las insuficiencias que poseia su conceptualización y medida, lo que se ha plasmado en opciones distintas que van desde las teorias sobre inteligencias múltiples (Gardner, 1983, 1993; Pelechano, 1989, 1996, 2000) hasta la propuesta triárquica de Stemberg $(1985,2003)$ y que incorporó poco después la posibilidad de otros tipos de competencias acerca de los procesos de cognición social. La tercera, que arrancó como una crítica de los modelos multifactoriales de la personalidad se ha plasmado en lo que se denomina teorías legas dentro de la psicología (Clayton \& Birren, 1980; Holliday \& Chandler, 1986; Sternberg, 1985).

Originalmente las teorías legas pretenden apresar lo que las personas no especialistas en psicología poseen y utilizan respecto a parcelas del funcionamiento psicológico tales como la inteligencia, la 
personalidad o la motivación. En buena cuenta, todos los humanos debemos tener algunas ideas acerca de cómo funcionamos nosotros mismos y los demás y gracias a esas ideas y modo de poner esas ideas en práctica nos relacionamos. Uno de los instrumentos más utilizados en el establecimiento de esas relaciones es el lenguaje, por lo que el estudio de la manera de utilizar y relacionar los términos lingüísticos en el habla de uso común, representará uno de los procedimientos que puedan utilizarse para estudiar la manera de entender la realidad y, específicamente, la realidad psicológica.

Repárese que, en esta acepción de "teoría lega", se estudia la manera de utilizar y relacionar las expresiones lingüisticas relacionadas con una temática dada. No trata de sustituir a los "modelos científicos" asentados en un discurso científico tradicional, sino de complementarlos en la medida en que este acercamiento lego reflejaría los límites y relaciones percibidas por los humanos no especialistas en un campo semántico dado. Y serían un marcador acerca de lo que las personas entienden por esa parcela de la realidad psicológica. Cuando no existen modelos científicos claros respecto a un campo de estudio, la apelación a las opiniones de las personas no especialistas puede iluminar unas primeras formulaciones 0 , alternativamente, mostrar el estado de límites difusos que presentan ciertos campos de estudio y posibilitar un estudio más profundo a partir de los resultados alcanzados.

Usualmente se presentan los resultados correspondientes a estas teorías legas como elementos comunes a todos los seres humanos de modo que existiría "una" teoría general y común. En la medida, sin embargo, en que se trate de parcelas del funcionamiento psicológico con fuerte arraigo y compromiso social, es posible encontrar distintas formulaciones legas en distintos contextos de vida. En una ocasión anterior (Pelechano y González Leandro, 2004) presentamos resultados de adolescentes y jóvenes en los que podian rastrearse cambios en la agrupación de los mismos términos desde los 14 a los 18 años. No existen, en español y con muestras españolas, estudios sobre este tema con muestras de adultos, situación que pretendemos corregir con este trabajo.

Pelechano (1990, Pelechano, Fumero y Garcia, 2004) sugirió una estrategia alternativa a las teorías legas, que se ha plasmado en 
resultados concretos y que consiste en acudir a los depósitos "populares" de conocimientos sobre un tema. En el caso de la sabiduría, uno de estos depósitos se encuentra en el cuerpo de refranes españoles. No se trata en este caso de un análisis de las relaciones entre términos lingüísticos sino de un considerable cuerpo de conocimientos decantados históricamente, utilizados en comunicación verbal durante varios siglos y que formarian una parte relevante de la sabiduría popular (calificada en más de una ocasión como filosofía del pueblo llano) de nuestra tradición cultural. Refranes, proverbios, adagios y decires existen en todas las lenguas conocidas y representan un cúmulo de conocimientos no siempre fáciles de entender y utilizar. Cuando se presentan ante los ciudadanos de nuestro tiempo, muchos de estos refranes son entendidos (aunque no se utilicen en el lenguaje cotidiano) y representan prescripciones eufónicas que exigen un tipo de conocimiento distinto al que se encuentra en los tests de inteligencia para que estos conocimientos sean aplicados de manera correcta.

En este trabajo se utilizará la primera y más extendida acepción de teoría lega o implícita. En el dominio de la sabiduría una de las primeras publicaciones fue la de Clayton y Birren (1980), que se desarrolló en dos etapas: en la primera se pidió a los participantes que generaran una lista de descriptores (preferiblemente adjetivos) que identificaran a las personas sabias. Posteriormente se pidió que los participantes calificaran la similitud entre estos descriptores. Los análisis evolutivos mostraron diferencias entre la manera de entender "sabiduría" a lo largo de la edad adulta. Sternberg (1985) encontró diferencias sobre modos de agrupación de descriptores de sabiduría en distintos grupos profesionales y Takahashi y Bordia (2000) encontraron diferencias asimismo en distintos contextos culturales como India, Estados Unidos, Australia y Japón. En este trabajo, se han empleado expresiones utilizadas por estos últimos autores y se intentará ofrecer, además de una consideración evolutiva, una cierta comparación transcultural.

En la medida en que las culturas orientales (India, Japón) y las occidentales (Australia, Estados Unidos) presentan resultados distintos, suponemos que los resultados correspondientes a España pueden situarse entre los dos polos dado el mestizaje cultural que caracteriza a nuestro país. 
Estos son los objetivos que se persiguen aqui: (a) presentar los resultados acerca de la concepción lega de sabiduría, inteligencia y anciano en adultos españoles; (b) utilizando procedimientos de análisis que permitan una comparación con resultados de otros países, ofrecer similitudes y diferencias y (c) estudiar las relaciones entre el yo real, el yo ideal y la sabiduría que acercaría los resultados alcanzados, a los estudios de los valores sobre este tema.

\section{MÉTODO}

\section{Participantes}

Han participado un total de 300 adultos, la mayoría, estudiantes universitarios de la Universidad de La Laguna, con una media de edad de 23,50 años (desviación típica de 6,8), un 65\% mujeres. La colaboración en el estudio ha sido voluntaria y la cumplimentación de las tareas llevaba consigo un pequeño incremento en la calificación final, en el caso de haber aprobado previamente la materia de psicología de la personalidad. Se han formado dos grupos ( $>$ y $<$ de 30 años) de edad cronológica) con el fin de poder llevar a cabo análisis transversales evolutivos. El número de participantes ha sido similar en los dos grupos de edad (21) y ello ha estado determinado por el número de participantes mayores de 30 años; el número de $<30$ años se ha obtenido mediante una extracción aleatoria del subgrupo de edad correspondiente (que era mucho más numeroso).

\section{Tarea a realizar}

Se entregaba a cada participante una hoja en la que se encontraban los 14 descriptores siguientes: con experiencia, intuitivo/a, introspectivo/ a (callado/a), pragmático/a (con sentido práctico), comprensivo/a, benévolo/a (amable), inteligente, pacifico/a (tranquilo/a), informado/a (erudito/a), con sentido del humor, observador/a (perspicaz), anciano/ a, yo mismo/a, sabio/a.

Se explicaba que la tarea consistía en comparar cada par de adjetivos y calificar su grado de "cercanía" o similitud en una escala de iba de 1 (nada que ver entre ellos) a 7 (son prácticamente lo mismo). 
Una vez terminada la tarea de comparación, se pedía que eligieran los tres descriptores (de entre los 14) que mejor reflejaran su "yo ideal" y que los ordenaran por importancia ( 1 la expresión que se ajustara más a su yo ideal; luego 2 , como la segunda y 3 respecto a la tercera).

\section{Procedimiento de recogida de información}

La tarea se realizó en pequeños grupos o de forma individual, a lo largo de dos meses. Se pedía a los participantes que no comentaran con sus compañeros nada referido a la tarea y a la manera de completarla. Finalmente, puesto que esta tarea formaba parte de un programa de prácticas voluntarias, los participantes cornpletaron otras pruebas y se les dio la posibilidad de tener información individual respecto a la significación de las puntuaciones que habian obtenido en todas las pruebas, pasado dos meses de haberlas cumplimentado y mediante entrevista personal con los profesores de la materia.

\section{RESULTADOS}

Puede resultar iluminador una anotación acerca de los procedimientos de análisis utilizados para la obtención de los resultados que se van a presentar. Las respuestas recogidas en la parte correspondiente a la calificación de similitud entre términos han sido analizadas con escalamiento multidimensional, con el programa ALSCAL del SPSS-12. Siguiendo a Kruskal y Wish (1978 y Coxon (1982) se ha utilizado el análisis de conglomerados para la interpretación del escalamiento multidimensional (criterio de similitudproximidad). Con el objetivo de encontrar resultados más interpretables, los resultados de los conglomerados fueron rotados a varimax $y$, posteriormente, los resultados obtenidos se sometieron a análisis jerárquico de conglomerados con el fin de ordenar los resultados por niveles de similitud: el criterio de agrupación utilizado ha sido la puntuación media de similitudes entre los grupos de términos, utilizando como medida el cuadrado de la distancia euclídea entre conceptos o grupos. En cada estadio de agrupamiento jerárquico se medía la distancia entre conceptos o grupos y se agrupaban los conceptos o grupos con menor distancia. De esa manera los elementos que se 
combinan en el primer estadio se encuentran más cercanos entre sí que los que se combinan en el segundo estadio, y así sucesivamente.

Por lo que se refiere a la elección de adjetivos que representen el yo-ideal, se pidieron tres y se dio una puntuación de 3 para el primero, 2 para el segundo y 1 para el tercero. Los resultados de estas puntuaciones se asignaron a cada término elegido y se calculó el porcentaje de puntuación que correspondía a cada uno de los términos.

En la medida en que el rango de edad lo permitió, se llevaron a cabo análisis por separado para el grupo de menos de 30 años y de más de 30 años, además, del grupo total.

\section{La "significación lega" de sabiduría, Inteligencia y sí mismo/a...}

Parecen existir tres tipos de tradiciones claras en la historia del pensamiento respecto a la significación general de "sabiduría". Una de ellas recogería un tipo de conocimiento especial que se refiere a la estructura formal del universo (en el caso de la metafísica) o de Dios (en el caso de la teología); sabiduría, en este sentido, se encuentra alejada de las acepciones "legas" y recogería las tradiciones, no especialmente consensuadas, de la metafísica. En otra acepción se hace depender la sabiduría de la eficiencia y la eficacia sociopersonal, en la solución satisfactoria de los problemas fundamentalmente tratados por las ciencias naturales $y$, de rechazo, por algunas ciencias sociales (lo que parece característico de una tradición occidental y que acercaría "sabio" a erudito, conocedor, observador y perspicaz). En la tercera tradición, de corte más oriental, la sabiduría tiende a unirse a elementos humanistas de pacifismo, comprensión y cordialidad, lo que se encontraría representada por expresiones tales como amable y comprensivo. Éstas dos últimas acepciones se encuentran representadas en las palabras elegidas en este estudio y que recogen las expresiones más usuales empleadas en estudios publicados sobre el tema. Para los objetivos que se persiguen en este trabajo, resultan de especial interés, las agrupaciones que ofrezcan las expresiones "sabio", "inteligente" y "anciano" en la medida, además, que existe una tendencia a asimilar "sabio" con edad avanzada, lo que se encuentra recogido tanto en la propuesta original y pionera de Jung como en la de Erikson. 
El resultado correspondiente a los conglomerados jerárquicos para toda la muestra de adultos se encuentra recogido en la figura 1 . La solución ha tenido un estrés de 0,22 y una $R^{2}=0,74$.

Figura 1.- Conglomerados jerárquicos en espacio rotado en 300 adultos españoles. La explicación en el texto

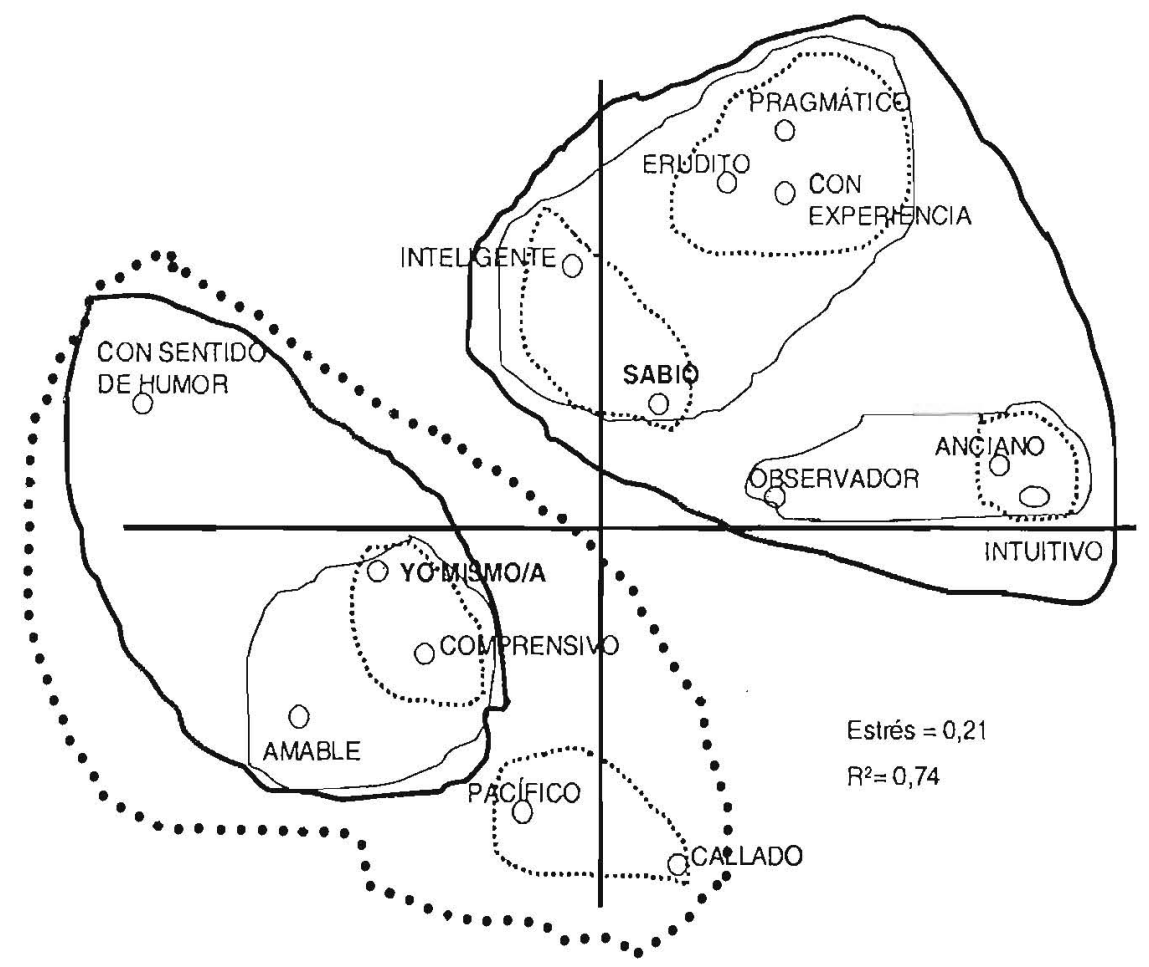

La primera "jerarquía" se encuentra bastante diversificada y forma cinco núcleos significativos: anciano-intuitivo, pragmático-con experiencia, inteligente-sabio, pacífico-callado y comprensivo-yo mismo. Dos de los tres primeros se encuentran totalmente ubicados en el primer cuadrante y los otros dos prácticamente en el tercero ${ }^{1}$. De las tres primeras agrupaciones observadas llama la atención la similitud percibida entre sabio/a-inteligente, anciano/a-intuitivo/a y

\footnotetext{
1 Esta distribución sugeriría que se podria interpretar la mayor parte de los resultados como un eje situado a $45^{\circ}$ del primero y tercero (posiblemente rotando los ejes actuales podría encontrarse una solución fundamentalmente unidimensional, aunque con una funcionalidad
} 
erudito/a con pragmático y con experiencia. Sugeriría que sabiduría e inteligencia poseen significaciones psicológicas similares, que el anciano es a la vez intuitivo (lo que no deja de sorprender y explicable, posiblemente, apelando a contextos en los que no se trata de temáticas o cuestiones nuevas sino de asimilar lo nuevo a algo ya conocido $y_{\text {, }}$ por ello, de explicarlo apelando a esos argumentos y contextos "ya vividos") y que el conocimiento que lleva a erudición se entiende en sus correlatos pragmáticos y experienciales. El referente del yo-real (yo mismo/a) se asimila a ser comprensivo/a en primer lugar (alejado de inteligente, erudito y anciano) y, finalmente, el ser pacífico/a se asimila a ser callado/a (esto es, a introversión).

El conglomerado de "anciano/a-intuitivo/a", situado en el primer cuadrante se agrupa en un segundo nivel con "observador" lo que da una imagen un tanto contradictoria (observador e intuitivo), aunque podria ser resuelta esta aparente contradicción por la referencia a situaciones "ya vividas" y, por ello, apreciación de lo relevante en las cuestiones de vida o de ciencia. Podría interpretarse como la observación y el "darse cuenta" del anciano, en el sentido de que se refiere a una persona que muchas veces se da cuenta de cosas sin apenas esfuerzo, simplemente debido a la edad ( $y$ lo que las experiencias de la edad llevan consigo).

El segundo conglomerado de primera jerarquía lo forman "pragmático/a", "erudito/a"(conocedor de muchas cosas) y "con experiencia". En un primer momento del análisis recogería la idea de que la erudición se asimila a un conocimiento grande y entresacado de la experiencia, aunque con una orientación práctica.

El tercer núcleo asimismo de primera jerarquía lo forma una dualidad que, aparentemente, debería diferenciarse pero que se percibe como un único conglomerado de primer nivel: "inteligente" y "sabio". Teórica y académicamente se diferencian con cierta claridad (la inteligencia se ocuparia de problemas "científicos" y la sabiduría de "problemas de vida") aunque los adultos, en general, perciben ambas expresiones con una significación similar.

dispar en la que anciano/a, sabio/a y erudito/a se encontrarían en el polo "positivo" mientras que yo mismo/a y la mayorla de expresiones referidas a funcionamiento humano cordial y facilitador de la interacción personal, se encontrarlan ubicados en el poto "negativo" u opuesto al anterior. $\mathrm{O}$, para simplificar, los elementos afectivo-positivos estarían en un polo (incluyendo yo mismo) y los cognitivos (incluyendo anclano/a) en el otro. 
Estos dos últimos conglomerados de primera jerarquỉa forman un conglomerado de mayor generalización y que recogería aquella tradición a la que hemos hecho referencia más arriba de sabiduría como inteligencia pragmática y con gran volumen de conocimientos. $Y$, de rechazo, sugeriría que inteligencia y sabiduría se referirian a elementos experienciales y pragmáticos, con una exigencia de posesión de muchos conocimientos. $Y$, alternativamente, que el valor pragmático de conocimientos y la experiencia se asimilan más con la inteligencia y la sabiduría que con anciano/a, intuitivo/a y observador/a.

Estos conglomerados, a su vez, se agrupan en otro más genérico en el que se integran los ocho marcadores de sabiduria que cubren prácticamente el primer cuadrante de la solución y que estaría formado por dos "ejes" que recogen lo que se acaba de mencionar: uno en el que "sabio-inteligente" serían los descriptores más relevantes y en el otro (relativamente independiente de éste) en el que se encontraría observador-intuitivo-anciano. La ubicación general de los dos conglomerados (primero y tercer cuadrante) indicaría que ambos poseen una significación funcional "opuesta". De hecho, se presentan como "independientes", sin que exista solapación alguna entre ellos. Esta dualidad de conglomerados no se encuentra entre los resultados transculturales de Takahashi y Bordia (2000), que, en cada caso han sido analizados siguiendo los mismos procedimientos que los que hemos utilizado nosotros.

Al conglomerado formado por yo mismo/a-comprensivo se añade "amable" en un segundo nivel, lo que sugeriría que independientemente o al margen de lo que se ha venido presentando, la imagen del sí mismo/ a que se posee se asimila a descriptores de buena relación interpersonal $y$, posteriormente, en un mayor grado de generalidad, se incorpora "sentido del humor", lo que acercaria la imagen del yo en este grupo a una cierta extraversión. El polo "introvertido" (pacífico/a-callado/a) se incorporaría en un último nivel de generalización a este número "personal", formando el segundo conglomerado jerárquico global.

\section{¿Cambio alrededor de los 30 años?}

Se han formado dos grupos de edad, uno de menos de 30 años y otro de más de 30 años. El criterio de asociación ha sido el supuesto 
de que alrededor de los 30 años, se encuentra la edad real de independencia económica de los padres. En la medida en que el número de participantes de más de 30 años ha sido escaso (21) se ha elegido, al azar, un número similar correspondiente a menos de 30 años con el fin de que las comparaciones entre ellos no estuviesen sesgadas por el volumen de participantes.

En el caso de participantes con edad menor a 30 años, los resultados correspondientes al estudio de conglomerados jerárquicos se encuentran representados en la figura 2.

Figura 2.- Conglomerados jerárquicos en espacio rotado. Adultos $<30$ años. La explicación en el texto

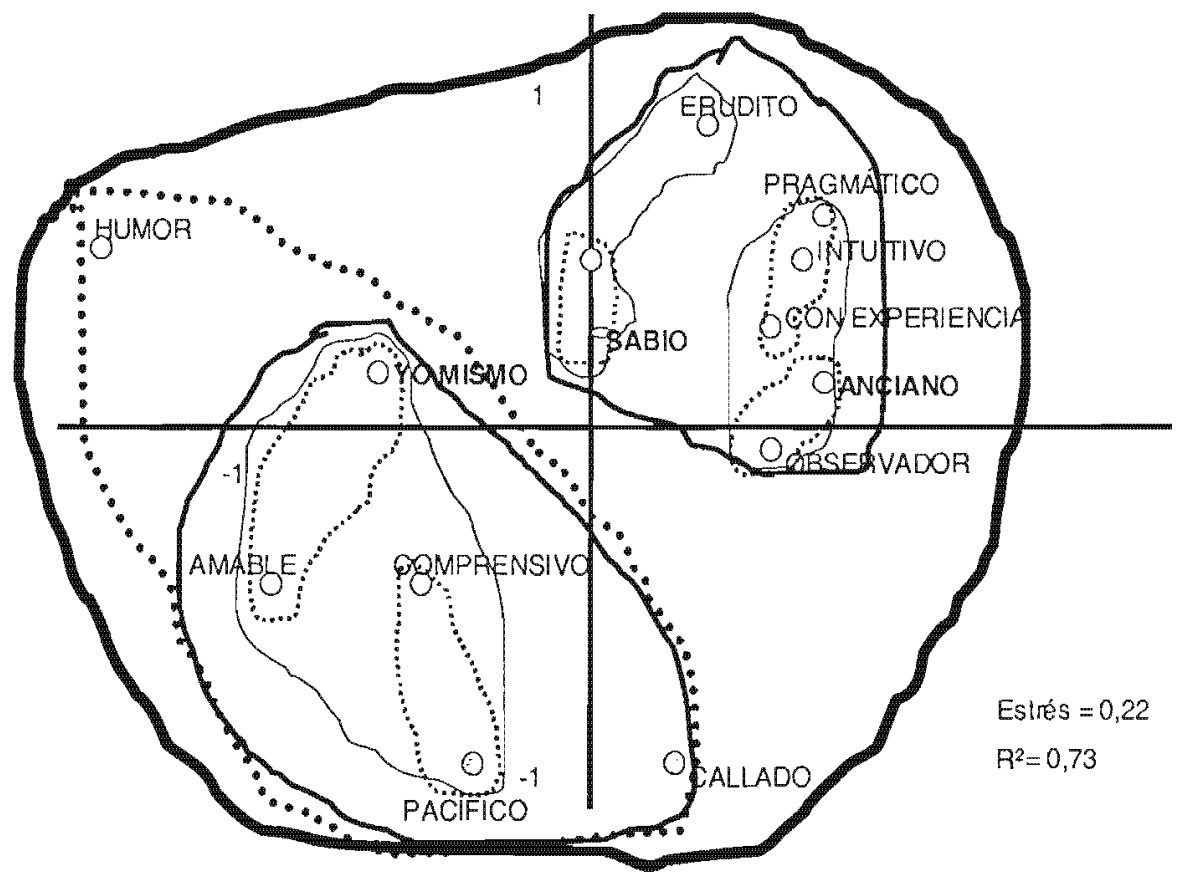

La solución en espacio bidimensional ha tenido un estrés de 0,22 y una $R^{2}=0,73$. En lineas generales se obtienen soluciones similares a las observadas en el grupo en general si bien, con alguna cualificación: de entrada hay que decir que todos los descriptores se agrupan, al final, en un único conglomerado, lo que hablaría de una menor diferenciación entre ellos para esta muestra más joven. 
En un primer agrupamiento jerárquico aparecen cinco agrupaciones de primer orden: (1) inteligente-sabio/a, (2) pragmático/a-intuitivo (frente a erudito/a en el grupo total)-con experiencia, (3) anciano-observador (intuitivo/a en el grupo total), (4) comprensivo/a-(callado/a en el grupo total)-pacífico/a y (5) yo mismo-amable (comprensivo/a en el grupo total). Repárese que solamente se mantiene el conglomerado formado con la asimilación de sabio con inteligente, respecto al grupo total.

En el primer cuadrante, un segundo nivel de generalización jerárquica, anciano/a-observador/a se une con pragmático/a-intuitivo/a-con experiencia, lo que sugeriría un dominio de conocimiento tanto fruto de la experiencia como del cultivo de la observación que se polarizaría alrededor de "anciano". Asimismo en ese mismo primer cuadrante el primer agrupamiento de sabio-inteligente se une a erudito (lo que formaría un segundo nivel jerárquico) y sugiere la idea de que sabio se une a persona erudita e inteligente. Estos dos agrupamientos de segundo nivel se agrupan en un tercero en el que sabio, inteligente y anciano van juntos. Llama la atención que el concepto "yo mismo" se encuentra un tanto alejado de estos agrupamientos, tal y como sucedía en el grupo total.

El agrupamiento de primer nivel yo mismo/a-amable se une con el otro de primer nivel comprensivo-pacífico en un segundo nivel, lo que indicaría un dominio de humanismo y de asociación del yo con atributos positivos de corte pacifista, comprensivo y amable. En un tercer nivel se asocia con "callado" y, finalmente, en un cuarto nivel con "sentido del humor".

Parece, de nuevo, que los elementos de corte más sentimentalafectivos y de interacción personal positiva forman un bloque, al que tiende a "oponerse" un conglomerado de cualidades de corte intelectualcognitivo en el que se encuentran sabio, anciano e inteligente. A diferencia de lo que ocurría en el análisis de toda la muestra $(\mathrm{N}=300)$, los dos grandes conglomerados se unen en un cuarto y último nivel que los integra. Desde aquí cabría pensar que todos los descriptores empleados se consideran relevantes y guardarían una cierta relación entre sl de inclusión, de unos respecto a otros, a través de agrupamientos de progresiva generalización.

En la figura número 3 se encuentra la representación del análisis de conglomerados jerárquicos y rotados en el grupo de más de 30 años, con un estrés de 0,22 y una $R^{2}=0,72$. 
Figura 3.- Conglomerados jerárquicos en espacio rotado. Adultos $>30$ años. La explicación en el texto

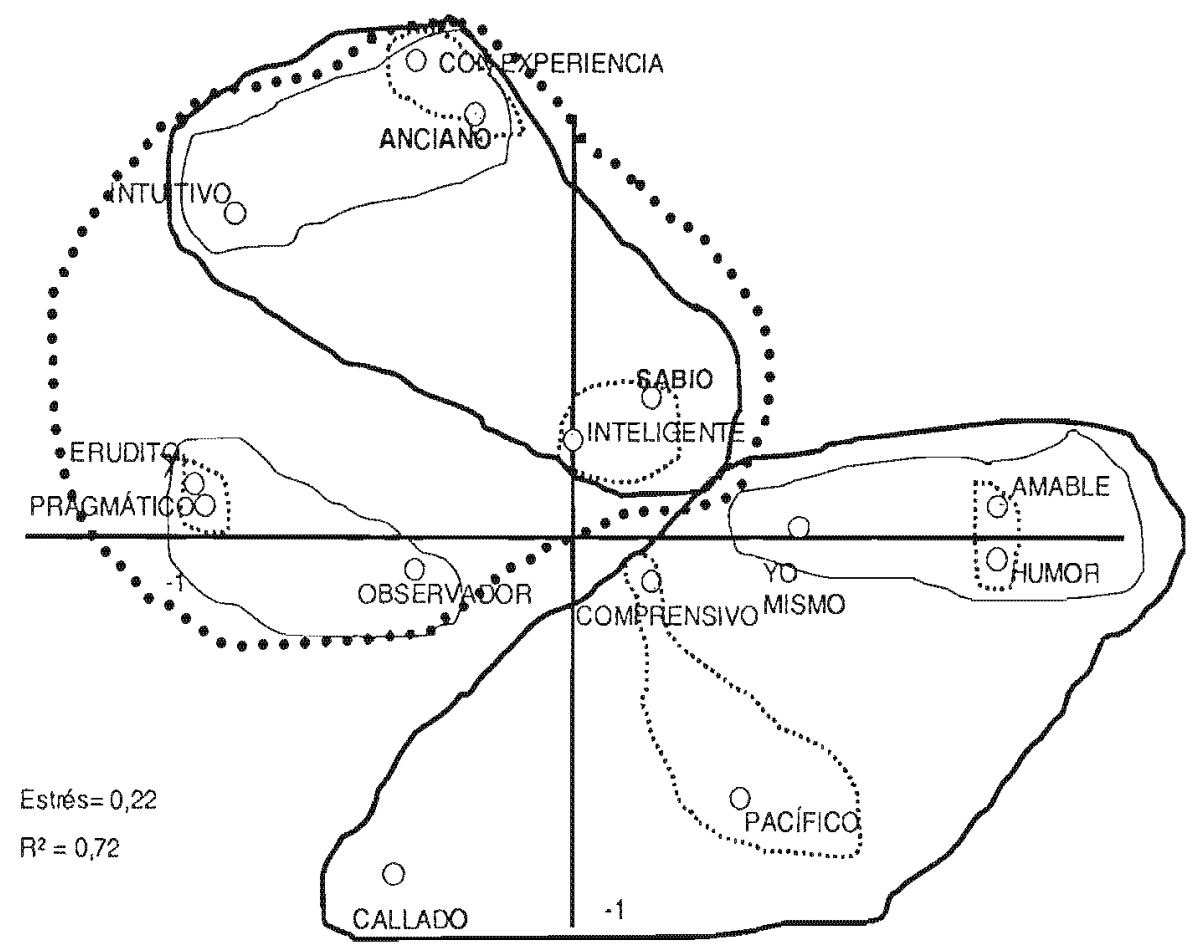

En una primera consideración habria que señalar que los resultados son iguales a los obtenidos en el grupo total, con alguna diferencia entre la que habría que señalar que ha habido una rotación hacia la derecha en el sentido de las agujas del reloj por lo que se refiere a la ubicación de los conglomerados aislados.

Se mantiene la conjunción entre sabio/a-inteligente en un primer nivel; asimismo, anciano/a se une con experimentado/a (frente a observador/ a que se encontraba en el grupo más joven). La erudición se entiende como un conocimiento pragmático que se une posteriormente a la observación. Todas estas apelaciones y calificativos se aúnan forman un conglomerado más general que podría caracterizarse como características y procesos psicológicos comprometidos con la inteligencia, sabiduría y la percepción de anciano/a. 
El segundo gran conglomerado se encuentra situado en el lado opuesto (cuarto cuadrante fundamentalmente en este caso) y en él se encuentran dos de primer nivel: (1) amable-con sentido del humor por una parte y (2) comprensivo-pacífico. Un segundo nivel de conglomerado se forma al integrar "yo mismo" al par amable-con sentido del humor $y$, en un tercer y último nivel, se añade "callado" a todos ellos. Este agrupamiento en general, recogería asimismo elementos humanistas-afectivos, en el que predominan características de extraversión y cordialidad referidos a la percepción que los participantes poseen de si mismos, con un marcador de introversión (callado/a).

Estos resultados parciales apuntarian a que en la acepción lega, sabio e inteligente van juntos, que en un nivel más general a ello se uniría anciano y que parece que existe una tendencia a diversificar los dominios psicológico-semánticos con la edad de manera que "sabio" se tiende a unir con acepciones intelectual-cognitivas mientras que el "yo mismo" lo hace con aspectos sentimental-humanistas.

Y, por otro lado, los resultados de los dos grupos de edad sugieren que después de los 30 años, los adultos tienden a presentar una mayor diferenciación entre elementos afectivos y cognitivos y que mientras los primeros tienden a atribuirlos a las características personales que les identifican, toda vez que los elementos que se refieren a procesos cognitivos no son utilizados como referentes de sí mismos (por decirlo de algún modo, parece que estos procesos y fenómenos se utilizan para enjuiciamientos "objetivos" y pragmáticos).

\section{La imagen del yo ideal, criterios de eficacia y de humanismo}

Se ha pedido en un segundo momento a los participantes que eligieran tres de las expresiones que acababan de calificar y que se encontraran más cerca del "yo ideal" que tuviesen para ellos mismos. En la medida en que se encontraba la expresión "yo mismo(a)" entre las expresiones, se podría entresacar, asimismo, el grado de coherencia entre la percepción de la imagen ideal que tendrían de sí mismos y la imagen real. Se recodificaron los resultados tal y como se ha expuesto más arriba y en la tabla 1 se encuentran los porcentajes de respuesta (modulados por importancia) que han asignado los 
participantes en total $(\mathrm{N}=300)$ y en los dos grupos de edad (reducidos los participantes en cada grupo a $\mathrm{N}=21$, para poder equilibrar la interpretación de las respuestas.

Tabla 1.- Valoración de descriptores del yo ideal en adultos españoles (porcentajes ponderados $<5 \%$ en general y en dos grupos de edad. La explicación en el texto)

\begin{tabular}{llll}
\hline PALABRAS & $\%$ & $\%<30$ & $>30$ \\
& TO- & AÑOS & AÑOS \\
& TAL & & \\
\hline Inteligente & 16,8 & 16 & 17,6 \\
Comprensivo/a & 15,4 & 12,7 & 14,8 \\
Con sentido del humor & 11,3 & 12,7 & 11,8 \\
Observador(a)/perspicaz & 7,1 & 9,5 & 4,3 \\
Sabio/a & 7 & 0 & 12,9 \\
Benóvolo(a)/amable & 6,7 & 11,7 & 5,5 \\
Con experiencia & 6,25 & 8,5 & 7 \\
Pragmático/sentido práctico & 6,25 & 2,1 & 9,4 \\
Yo mismo(a) & 6,1 & 12,7 & 5,8 \\
Pacífico(a)/tranquilo(a) & 5,6 & 9,5 & 5,7 \\
Intuitivo/a & 5 & 0 & 3,7 \\
Introspectivo(a)/callado(a) & 3 & 4,2 & 2,3 \\
Informado(a(/erudito(a) & 2,9 & 0 & 0 \\
Anciano(a) & 0,2 & 0, & 0 \\
\hline (la diferencia en "anciano" se debe a que en las & & & \\
submuestras seleccionadas no aparece ni una & & & \\
elección (N=21 en sujetos que seleccionados & & & \\
para análisis por grupos de edad) & & & \\
\hline
\end{tabular}

Nota: porcentajes menos al $5 \%$ en elección en grupo total: introspectivo(a)/callado(a): $3 \%$; informado(a): $2,9 \%$; Anciano(a): $0,2 \%$.

Mientras los resultados presentados hasta aquí se refieren a las afinidades percibidas "fácticas" entre los conceptos, los que vamos a ofrecer ahora se mueven en el mundo de los deseos, de las aspiraciones e incluso de la "mejora" ideal como ser humano respecto a una serie de referentes entre los que se encuentra sabiduría y otros procesos cognitivos, frente a otro núcleo conceptual que recogería caracteristicas de cualidades humanas positivas.

El primer gran resultado que debe tomarse en consideración es que no existe ningún concepto que identifique, mayoritariamente, los deseos de "yo ideal": para la muestra total el término con mayor porcentaje es "inteligente" y no llega al $20 \%$; algo similar sucede en los 
caso de las dos muestras parciales. Ello significaria que existe una considerable dispersión respecto a la imagen de "yo ideal" que persiguen los adultos.

Un segundo resultado de gran interés es el que se refiere al grado de aceptación del sí mismo ("yo mismo") y/o su congruencia con el yo ideal. El porcentaje ponderado de elección como marcador de ese yo ideal es el $6,1 \%$. Sería un indicador de que no tiende a ser frecuente la aceptación de sí mismo/a, ni de una actitud conformista, ni de una coherencia entre cómo se es y cómo se desea ser. Se puede rastrear que existe una mayor aceptación en la submuestra más joven y que esa aceptación disminuye cuando se pasan los 30 años de edad.

En la medida en que se pedía la elección de tres descriptores, los tres primeros en elección representarian mayoritariamente las tres características más perseguidas (las puntuaciones máximas, en el caos de tratamiento "igualitario" deberían ser del 33,3\% en cada elección). Esta "trilogía" de perfección ideal, aunque alejada del $99,9 \%$ de elección, estaría formada por un atributo cognitivo (inteligente) y dos a mitad de camino entre eficacias cognitivas y emocionales (sentido del humor y ser comprensivo/a), todos ellos con un índice cuantitativo superior al $10 \%$. Este resultado tiende a mantenerse a todo lo largo de la edad estudiada aunque existen unas ciertas disonancias moduladas por al edad en otros descriptores (la benevolencia se valora más en el grupo más joven).

A diferencia de lo que sucede en el trabajo de Bordia y Takahashi (2000), ser "sabio/a" representa una elección que se encuentra en un nivel intermedio de deseo en la muestra general y se detecta una diferencia clara con respecto a la edad: los de menos de 30 años no desean para nada ser sabios, lo contrario de lo que sucede con los de más de 30 años. En la medida en que sabio se ha unido a inteligente en el análisis de conglomerados y que "inteligente" representa una elección poderosa en todas las edades, nos encontramos con una disonancia en este tipo de resultados y que podría significar, en todo caso, que se desea ser inteligente pero no sabio. Tanto más cuanto que sabio tiende a unirse con "anciano" (la expresión de yo ideal menos elegida de todas las propuestas).

Un bloque que se encuentra entre el 5 y el 9,5 por 100 de elección lo forman las expresiones de "observador/a", "benévolo(a)/amable", "con 
experiencia", "pragmático(a)/con sentido práctico" y "pacífico(a)/ tranquilo(a)". Se trata de una mezcla de capacidades cognitivas y afectivas, todas ellas con un referente aplicado.

Junto a anciano, la expresión menos elegida es la de "informado(a)/ erudito(a)n . Se diferencia claramente entre inteligente y erudito y se toma partido por lo primero. Queda por conocer cómo se operacionaliza claramente la inteligencia sin hacer mención al conocimiento de información amplio y serio. Tampoco, la intuición se encuentra especialmente valorada.

\section{CONCLUSIONES Y DISCUSIÓN}

Es verdad que este estudio no puede concebirse únicamente como una replicación del de Takahashi y Bordia (2000) en la medida en que se han incorporado términos nuevos y el análisis de conglomerados, obviamente, resulta sensible a la incorporación de dominios semánticos. Sin embargo, puede rastrearse que en la muestra más joven (básicamente universitaria, como en el caso de estos autores), todas las expresiones forman un conglomerado de orden superior (que se diversifica en dos cuando se pasa de los 30 años) y, por otro lado, que los resultados españoles tienden a situarse entre los de la India y los de Estados Unidos.

Sucede, además, que en cuanto se introduce la expresión "yo mismo", se observa una diversificación en dos tipos de conglomerados $y$, entre los dos, "sabio" se une a cualidades de orden cognitivo y de solución pragmática de los problemas, más que de cualidades de "buena persona", comprensiva y amable. La conclusión provisional que de esto se deriva es que, al menos en España, la idea de sabio se acerca a la de "experto", con conocimientos y cualidades intelectuales adecuadas para la solución de problemas concretos y de tipo pragmático. Los resultados que hemos alcanzado, además, sugieren que la cualidad que los adultos parecen valorar y es más llamativa, es la inteligencia que, curiosamente, en cuanto "valor", se diferencia mucho de "sabiduría" (no en cuanto a descriptores, pero sí en cuanto a deseo de logro). En lo que se refiere a pacifismo, comprensión de los demás, amabilidad e incluso sentido del humor tiende a ser percibido como un conglomerado de características personales a lograr. Los 
dos dominios (sabiduria-inteligencia y yo mismo) tienden a divergir con el paso de los años y de la experiencia personal acumulada.

Sucede, además, que no se desea ser sabio, ni tener erudición, sino únicamente ser inteligente, poder entender a los demás y tener sentido del humor. La inteligencia se asimila a ser experto en algo, entender a los demás unido a ser amable y cordial y el sentido del humor no está claro si se refiere a reaccionar con humor ante las situaciones de la vida, tener "gracia" para contar las cosas o percibir con humor la realidad que nos rodea (esto es, generar o percibir humor). En todo caso, la percepción y valoración de sabio y sabiduría en España tiende a ser bastante distinto de lo que sucede en Japón, India, Estados Unidos y Australia. Las limitaciones de la muestra utilizada recomiendan tomar estos resultados con precaución, pero la línea de demarcación respecto a diferencias en conglomerados jerárquicos y en asociaciones al yo ideal parece clara y demuestra que se trata de dominios ciertamente sensibles a las influencias culturales. En nuestro contexto, estas influencias nos sitúan un poco alejados de otros países y culturas.

\section{REFERENCIAS BIBLIOGRÁFICAS}

Clayton, V. \& Birren, J. E. (1980).- The development of wisdom across the life-span: A re-examination of and ancient topic. En P. C. Baltes \& O. G. Brian (eds).- Life-span development and behaviour, vol. 3, New York, Academic Press.

Coxon, A. P. M. (1982).- The user's guide to multidimensional scaling, London, Heinemann Educational Books.

Gardner, H.(1983).- Frames of Mind: The theory of multiple intelligences, New York, Basic Books.

Gardner, H.(1993).- Multiple intelligences. The theory in practice, New York, Basic Books.

Holliday, S. G \& Chandler, M. J. (1986).- Wisdom: Explorations in human competence, Basel, Karger.

Kruskal, J. B., \& Wish, M. (1978).- Multidimensional scaling, Newbury Park, CA, Sage.

Pelechano, V. (1990).- La psicología de los refranes: un recurso soslayado por l evaluación psicológica, Papeles del Psicólogo, 46/ 47, 37-49. 
Pelechano, V. (1996).- Una introducción al modelo de parámetros. En V. Pelechano (dir).- Psicología de la personalidad. I. Teorías, Barcelona, Ariel.

Pelechano, V., Fumero, A. \& García, L. (2004).- Refranes y sabiduría: el cuestionario REFPRUD-1 de Pelechano: teoría, lógica y datos de validación, Análisis y Modificación de Conducta, 30, 1-30.

Pelechano, V. y González-Leandro, P. (2004).- La concepción lega de sabiduría, inteligencia y envejecimiento en adolescentes, Análisis y Modificación de Conducta,30, 437-460.

Stemberg, R. J. (1985).- Implicit theories of intelligence, creativity and wisdom, Journal of Personality and Social Psychology, 49, 607-627. Sternberg, R. J. (2003).- Driven to despair: Why we need to redefine the concept and measurement of intelligence. En L. G. Aspinwall \& U. M. Staudinger (eds).- A Psychology of human strengths, Washington, American Psychological Association.

Takahashi, M. \& Bordia, P. (2000).- The concept of wisdom: A crosscultural comparison, International Joumal of Psychology, 35, 1-9. 


\section{FE DE ERRATAS}

Análisis y Modificación de Conducta, Vol. 32, № 141

Los autores del artículo "Teoría LEGA de sabiduría en adultos y relaciones con el Yo ideal" (pp. 65-83) son V. Pelechano, P. González-Leandro y L. García. 